\title{
Towards augmenting ambient systems networks and technologies
}

\author{
Haroon Malik ${ }^{1}$. Ansar-UI-Haque Yasar $^{2}$ - Elhadi M. Shakshuki ${ }^{3}$
}

Published online: 1 March 2018

c) Springer-Verlag GmbH Germany, part of Springer Nature 2018

This special issue is based on the best papers from the 8th International Conference on Ambient Systems, Networks and Technologies (ANT-2017), which was held in Madeira, a Portuguese archipelago, on 16-19 May 2017. The conference attracted a large number of scientific papers that contributed to the state-of-the-art in the ambient systems, networks and technologies. All the papers selected for this special issue have been extended from their original versions and underwent two rounds of rigorous peer-review process. Based on the reviewers' feedback, as well as the evaluations of the Guest Editors, nine papers were selected for this special issue from 13 invited submissions. The accepted papers augment ambient systems by uncovering interesting methodologies related to autonomic networks and communications, tracking and sensing technologies, system security and privacy, and intelligent computing and applications.

The first paper by El-Alfy et al. is entitled "Automated Gait-Based Gender Identification Using Fuzzy Local Binary Patterns with Tuned Parameters". The authors present a method for gait-based gender identification using a modified method of fuzzy local binary patterns to tune its hyperparameters. Texture features are extracted and used in classification using SVM with linear kernel. The proposed method is evaluated for normal-walk, carrying-bag and wearing-coat scenarios. Several performance measures are compared with four other variants of the local binary pattern (LBP) method.

Elhadi M. Shakshuki

elhadi.shakshuki@acadiau.ca

Haroon Malik

malikh@marshall.edu

Ansar-Ul-Haque Yasar

ansar.yasar@uhasselt.be

1 Weisberg Division of Computer Science, Huntington, WV, USA

2 Transportation Research Institute Hasselt University, Hasselt, Belgium

3 Jodrey School of Computer Science Acadia University, Wolfville, Canada
The experimental results show that the proposed fuzzy local binary pattern with tuned parameter (FLBP*) outperforms other methods for texture representation of gait energy images (GEI) in all tested scenarios. The highest performance is achieved for normal walking with accuracy above $96 \%$ and is more robust to variations in camera view angle as demonstrated by the tighter significance intervals.

The second paper by Iglesias-Urkia et al. is entitled "Analysis of CoAP Implementations for Industrial Internet of Things: A Survey". The authors first provides a general review of IoT. Then, deliver an overview of Constrained Application Protocol (CoAP). The CoAP protocol is lightweight and capable of running in resource constrained devices and networks and can be secured using Datagram Transport Layer Security (DTLS). The authors then present a feature and empirical comparison of several open source co-op implementations and also analyze the security libraries. The authors conclude that all the analyzed libraries but CoAPy have their use case. More heavyweight implementations can be used as clients in backbone systems as they are fast and those systems do not have a lack of resources; whereas, more lightweight libraries can be used in more resource constrained devices.

The third paper by Cai et al. is entitled "Tool-Supported Design of Data Aggregation Processes in Cloud Monitoring Systems". The authors proposed a systematic design approach for designing data aggregation in cloud monitoring systems. The approach relies on the systematic specification of data aggregation processes based on DAG-GTAX (Data AGGregation TAXonomy), and the SAT-based consistency check of the specification. The authors implemented SAFARE, a tool that supports the DAGGTAX-based specification, and integrates the Microsoft Z3 theorem prover to verify the specification formally. The proposed tool helps designers to design data management solutions for the data aggregation process (DAP), by guiding them to select proper DBMS transaction support for the DAP, and providing query struts for designing the database model and queries.

The fourth paper by Misek and Zavoral is entitled "Semantic Analysis of Ambiguous Types in Dynamic 
Languages". The authors presented an analysis of the PHP dynamic language source code while effectively maintaining a set of possible types for each variable and expression in the code. The cloud-based solutions still use legacy programming languages and technologies, such as the PHP language. For their effective integration into modern platforms, it is important to understand the legacy code base to provide modern analysis, testing and eventually compiler tooling. Towards this end, the authors describe a solution that combines known static language code analysis techniques, and that is enhanced by numerous modifications. The authors provide a design and implementation of a PHP dynamic language code analysis, compiler and runtime, which improves performance and allows for more advanced high-level code analysis and tools, all incorporated into the modern platform of .NET Core while taking advantage of the Microsoft Roslyn Compiler Platform.

Fifth paper by Ruta et al. is entitled "CoAP-based Collaborative Sensor Networks in the Semantic Web of Things". The authors described a semantic sensor network framework (SWoT) suitable for applications requiring advanced context detection and annotation, such as environmental monitoring and ambient intelligence. It is based on a backwardcompatible extension of the CoAP, supporting advanced semantic matchmaking via non-standard inference services. To evaluate the feasibility of the framework, the authors perform a case study in a hybrid sensor and vehicular network scenario and conducted experimental tests on a real testbed implementation.

Sixth paper by Anwar et al. is entitled "A Framework for Single and Multiple Anomalies Localization in Pipelines". The authors proposed an effective solution to monitor pipelines and provided a framework for anomaly localization using Cooja simulator and geographical information systems (GIS) that can also be used in pre-disaster management scenarios, i.e., pipelines can be maintained prior to actual leaks and spills. Timely precautionary measures can thus be taken during the pre-disaster, disaster and post disaster stages, thereby minimizing wastage of natural resources. Authors also compared localization accuracy with two detection and localization techniques namely: negative pressure wave (NPW) and pressure point analysis (PPA).

The seventh paper is Kamoun and Outay is entitled "IP/ MPLS Networks with Hardened Pipes: Service Concepts, Traffic Engineering and Design Considerations". IP hard pipe is a promising technology that allows carriers and service providers to fulfil the needs for guaranteed bandwidth, high availability, and a firm ultra-low end-to-end delay bound with no queuing loss to support their mission-critical services. In this context, the authors carried out an in-depth investigation of the traffic engineering and design considerations that need to be taken into account when deploying hard pipe strata in IP/MPLS networks. In particular, the authors focused on LSP provisioning, QoS management, time synchronization, availability, delay/jitter requirements and interoperability considerations. The authors also discuss some of the design and traffic engineering alternatives, critique their relative merits and propose some recommendations to enhance bandwidth usage, provide support for delivering end-to-end QoS and maximize revenues.

The eighth paper by Vega et al. is entitled "A Didactic and Interactive Language for the Construction of Adaptive Automata in Active Learning Environments". The authors designed an interpretable language called DInATon (Didactical and Interactive Adaptive Automata Construction Language). Authors considered several aspects when designing the DInAton language, such as emphasizing its interpretive characteristics, proximity to typical programming languages, mechanisms for textual and graphical representation of under construction automata. Although the DInAton language is still in the definition process, the authors found the results, so far produced by the DInAton language-based learning environment project, as promising. Currently, DInAton language supports the construction of finite automata and adaptive automata with certain topological constraints.

The ninth paper by Abutair et al. is entitled "CBR-PDS: A Case-Based Reasoning Phishing Detection System”. Phishing is a method in which attackers trick users into revealing sensitive information related to work, financial credentials, or even personal data to be used in fraudulent activities. The authors proposed a phishing detection system (CBR-PDS). The proposed system, i.e., CBR-PDS is based on a case based reasoning to be adaptive to any change in phishing behaviour, tactics, and techniques. It uses a two stage feature selection and weighting process to choose the relevant features and assign them appropriate weights. The reduction of the number of features amounts to a better accuracy and a reduced data dimensionality that tacitly improves the system processing time. The authors also did a comparative study of the performance of the proposed system with that of the most competitive classifiers.

The guest editors would like to take this opportunity to thank all the authors for the efforts they put in the preparation of their manuscripts and for their valuable contributions. We wish to express our deepest gratitude to the referees who provided very useful and thoughtful feedback to our authors. Our sincere thanks go to the Editor-in-Chief for his kind help and support. 